\title{
PROPAGATION OF AN ELECTROMAGNETIC SOLITON IN A FERROMAGNETIC MEDIUM
}

\author{
V. VEERAKUMAR ${ }^{1}$ and M. DANIEL ${ }^{1}$
}

(Received 10 May, 2000; revised 28 July, 2000)

\begin{abstract}
We study the propagation of electromagnetic waves (EMWs) in both isotropic and anisotropic ferromagnetic material media. As the EMW propagates through linear chargefree isotropic and anisotropic ferromagnetic media, it is found that the magnetic field and the magnetic induction components of the EMW and the magnetization excitations of the medium are in the form of solitons. However, the electromagnetic soliton gets damped and decelerates in the case of a charged medium. In the case of a charge-free nonlinear ferromagnetic medium we obtain results similar to those for the linear case.
\end{abstract}

\section{Introduction}

Electromagnetic wave (EMW) propagation through ordered magnetic material media has assumed lot of importance in recent times in the field of magneto optics [11]. In this direction, some time ago Nakata [7] and Leblond [6] separately studied the EMW propagation in a ferromagnetic medium without taking into account the exchange interaction in ferromagnets and found soliton excitations of magnetization of the medium. In this paper, we present the results of EMW propagation in an isotropic charge-free (both linear and nonlinear) as well as charged ferromagnetic material media by taking into account the basic spin-spin exchange interaction. Our study is based on the interaction of the magnetic field component of the EMW with the magnetization of the ferromagnetic medium. The dynamics of magnetization $\mathbf{M}(\mathbf{r}, t)$ in a ferromagnet in the classical continuum limit are in general governed by the Landau-Lifshitz (LL) equation with Gilbert damping [5]

$$
\mathbf{M}_{\boldsymbol{t}}=\mathbf{M} \wedge \mathbf{M}_{\mathrm{eff}}+\alpha \mathbf{M} \wedge \mathbf{M} \wedge \mathbf{M}_{\mathrm{eff}}, \quad \mathbf{M}^{2}=1,
$$

\footnotetext{
'Centre for Nonlinear Dynamics, Department of Physics, Bharathidasan University, Tiruchirapalli 620 024, India; e-mail: veera@kaveri.bdu.ernet.in.

(C) Australian Mathematical Society 2002, Serial-fee code 1446-1811/02
} 
where $\mathbf{M}_{\text {eff }}$ represents the effective field due to various magnetic interactions including interaction with the external magnetic field. Here the suffix $t$ represents partial differentiation with respect to time. To study the propagation of EMWs in any material media we have to consider Maxwell's equations describing the dynamics of the electromagnetic field [4].

$$
\begin{aligned}
\nabla \cdot \mathbf{E}=\frac{1}{\epsilon_{0}} \rho, & \nabla \cdot \mathbf{B}=0, \\
\nabla \wedge \mathbf{E}=-\frac{\partial \mathbf{B}}{\partial t}, & \nabla \wedge \mathbf{H}=\mathbf{J}+\epsilon_{0} \frac{\partial \mathbf{E}}{\partial t} .
\end{aligned}
$$

Here $\mathbf{E}=\left(E^{x}, E^{y}, E^{z}\right)$ is the electric field, $\mathbf{B}=\left(B^{x}, B^{y}, B^{z}\right)$ is the magnetic induction, $\mathbf{H}=\left(H^{x}, H^{y}, H^{z}\right)$ is the magnetic field, $\epsilon_{0}$ is the dielectric constant of the medium, $\rho$ is the charge density and $\mathbf{J}$ is the current density. For slowly-moving charges the current density given above takes the form $\mathbf{J}=\sigma \mathbf{E}$, where $\sigma$ is the conductivity of the medium. Using this and (2a) in the continuity equation for the free current given by $\nabla \cdot \mathbf{J}=-\partial \rho / \partial t$, we obtain $\rho(t)=\rho(0) \exp \left(-\sigma t / \epsilon_{0}\right)$. This shows that any initial free charge density $\rho(0)$ dissipates and therefore the first equation of (2a) takes the form $\nabla \cdot \mathbf{E}=0$. Now, taking curl on both sides of the second equation of (2b) and using the second relation of (2a) and the first of (2b) and after a little algebra, Maxwell's equations can be put in the form

$$
\nabla(\nabla \cdot \mathbf{H})-\nabla^{2} \mathbf{H}+\sigma \mathbf{B}_{t}=\mathbf{B}_{t} .
$$

Thus the set of coupled equations (1) and (3) completely describe the propagation of an EMW in a ferromagnetic medium and by solving them, we will obtain the nature of propagation of the EMW in the ferromagnetic medium and the magnetization dynamics of the medium. Therefore in the next section we solve them in the case of one spatial dimension for specific magnetic interactions.

\section{An electromagnetic soliton in an isotropic ferromagnetic medium}

A. Charge-free medium The effective field due to spin-spin exchange interaction and interaction with an external field in the case of an isotropic ferromagnet can be written as $\mathbf{M}_{\text {eff }}=\left[\nabla^{2} \mathbf{M}+2 A \mathbf{H}\right]$. When there are no free charges present in the medium the term proportional to Gilbert damping in (1) is irrelevant and hence the LL equation in this particular case $((1)$ when $\alpha=0)$ can be written as

$$
\mathbf{M}_{t}=\mathbf{M} \wedge\left[\hat{J} \nabla^{2} \mathbf{M}+2 A \mathbf{H}\right], \quad \mathbf{M}^{2}=1,
$$

where $A=g \mu_{b}, g$ is the gyromagnetic ratio, $\mu_{b}$ the Bohr magneton and $\hat{J}$ represents the exchange integral. Here $\mathbf{H}$ represents the external magnetic field which in our 
case is taken as the magnetic field component of the propagating EM field. In the case of untreated ferromagnetic materials, the magnetization $\mathbf{M}$, the magnetic induction $\mathbf{B}$ and the magnetic field $H$ are connected by the linear relation $\mathbf{H}=\mathbf{B} / \mu_{0}-M$, where $\mu_{0}$ is the permeability of the ferromagnetic material [4]. After using the above relation and dropping the term proportional to $\sigma,(3)$ can be written as

$$
c^{2} \nabla^{2} \mathbf{B}-\mathbf{B}_{u}=\frac{1}{\epsilon_{0}}\left[\nabla^{2} \mathbf{M}-\nabla(\nabla \cdot \mathbf{M})\right]
$$

Here $c=1 / \sqrt{\mu_{0} \epsilon_{0}}$ is the velocity of propagation of the EMW in the medium. Equations (4) and (5) govern the propagation of EMWs in a charge-free isotropic ferromagnetic medium. We solve the above equations in the one-dimensional case after making a multiple scaling and perturbation analysis $[2,12]$. For this we introduce the wave variable $\hat{\xi}=x-v t$ and stretch the wave and time variables by introducing $\xi=\varepsilon \hat{\xi}, \tau=\varepsilon^{3} t$, where $\epsilon$ is a very small parameter. We then expand $\mathbf{B}$ and $\mathbf{M}[2,12]$ uniformly as $\mathbf{M}=\mathbf{M}_{0}+\varepsilon \mathbf{M}_{1}+\varepsilon^{2} \mathbf{M}_{2}+\cdots, \mathbf{B}=\mathbf{B}_{0}+\varepsilon \mathbf{B}_{1}+\varepsilon^{2} \mathbf{B}_{2}+\cdots$, substitute them in the one-dimensional version of (4) and (5), coHect the coefficients of different powers of $\varepsilon$ and solve the resultant equations. The results at $O\left(\varepsilon^{0}\right)$ show that the magnetization $\mathbf{M}_{0}$ is restricted to the $(y-z)$ plane $(\phi=\pi / 2)$ and hence we choose $\mathbf{M}_{0}=(0, \sin \theta(\xi, \tau), \cos \theta(\xi, \tau))$. At $O\left(\varepsilon^{1}\right)$ we obtain $B_{1}^{x}=0, B_{1}^{\alpha}=k M_{1}^{\alpha}$ and $M_{1}^{x}=\left(\mu_{0} v / 2 A B_{0}^{z}\right)\left(\partial M_{0}^{y} / \partial \xi\right)$, where $k=\left[\epsilon_{0}\left(c^{2}-v^{2}\right)\right]^{-1}$ and $\alpha=y, z$. At $O\left(\varepsilon^{2}\right)$ after using the results of $O\left(\varepsilon^{0}\right)$ and $O\left(\varepsilon^{1}\right)$ (after lengthy calculations), we finally obtain (for details see [2])

where

$$
f_{\tau}+\frac{3}{2} \mu f^{2} f_{\xi}+\mu f_{\xi \xi \xi}=0
$$

$$
f=\frac{\partial \theta}{\partial \xi} \quad \text { and } \quad \mu=\frac{\mu_{0} k}{4 A v \epsilon_{0}}\left[\hat{J}-\frac{v^{2} k^{2}}{2 A}\right]
$$

Equation (6) is the well-known completely integrable modified Korteweg-de-Vries (mKdV) equation possessing $N$-soliton solutions [14]. For instance, the one-soliton solution of (6) can be written as $f=2 a$ sech $a \zeta$, where $\zeta=\xi-\eta \tau, a^{2}=\eta / \mu$ and $\eta=$ constant. Knowing $f, \theta$ can be calculated and hence we obtain the $y$ and $z$ components of magnetization at the lowest existing order as $M_{0}^{y}=1-2 \operatorname{sech}^{2} a \zeta$ and $M_{0}^{z}=$ $2 \tanh a \zeta \operatorname{sech} a \zeta$. Using the solution of $M_{0}^{y}$ in the relation for $M_{1}^{x}$ we obtain the $x$ component of magnetization at the lowest existing order as $M_{1}^{x}=\left(\mu_{0} a v k / A\right) \operatorname{sech} a \zeta$. From the values of $\mathbf{M}$ and $\mathbf{B}, \mathbf{H}$ can be calculated. The results show that the excitation of magnetization, the magnetic induction and the magnetic field are highly localized and appear in the form of solitons. To elucidate this we have plotted the components of the magnetization $M_{1}^{x}, M_{0}^{y}$ and $M_{0}^{z}$ in Figures 1 (a)-(c). 


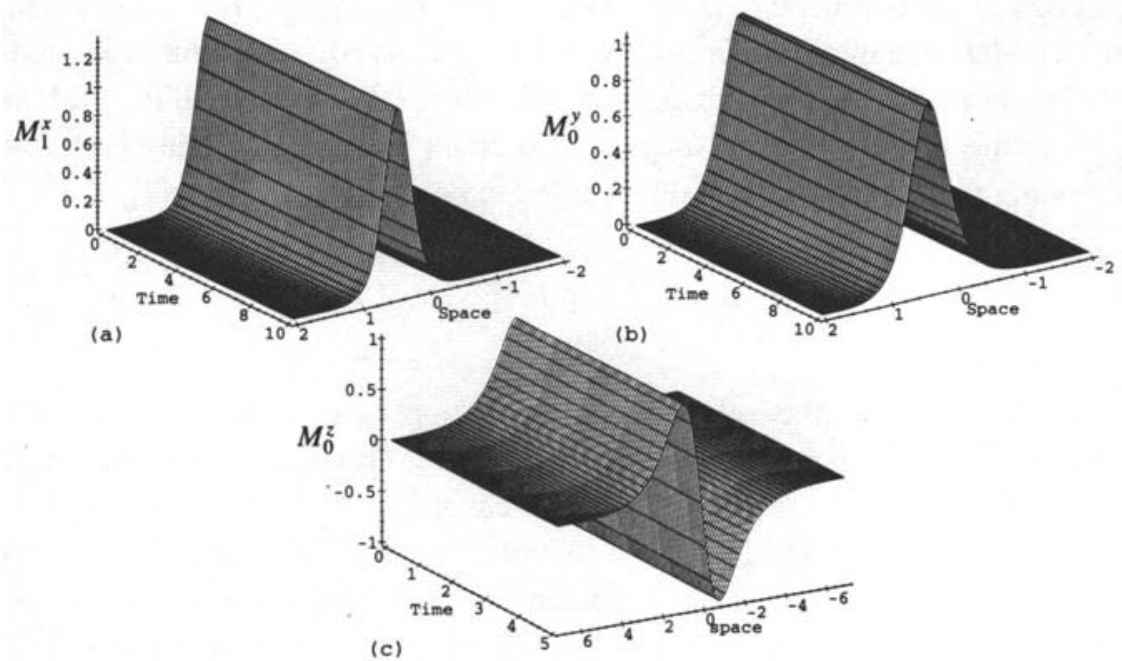

FIGURE 1. Soliton excitation of the magnetization (a) $M_{1}^{x}$, (b) $M_{0}^{y}$ and (c) $M_{0}^{z}$ in the isotropic charge-free ferromagnetic medium for $\eta=0.001, a=1$ and $A=-\mu_{o} v k$.

B. Medium with free charges When there are free charges present in the medium, one expects damping and the LL equation in this case should be the complete version of (1) with Gilbert damping.

$$
\mathbf{M}_{t}=\mathbf{M} \wedge\left[\hat{J} \nabla^{2} \mathbf{M}+2 A \mathbf{H}\right]+\alpha\left\{\mathbf{M} \wedge\left[\mathbf{M} \wedge\left(\nabla^{2} \mathbf{M}+2 A \mathbf{H}\right)\right]\right\}, \quad \mathbf{M}^{2}=1 .
$$

Now the set of coupled equations (3) and (7) describe the EMW propagation in the ferromagnetic medium with free charges. Following the same procedure as in the case of charge-free medium [12], by choosing the conductivity $\sigma$ as $\sigma=\varepsilon^{3} \sigma$ and the damping parameter as $\alpha=\varepsilon \alpha$, after lengthy calculations we end up with a perturbed $\mathrm{mKdV}$ equation with a dissipation term $\alpha J\left(\partial^{2} f / \partial \xi^{2}\right)$, which upon making the transformations $f^{\prime} \rightarrow f / 2$ and $\tau^{\prime} \rightarrow \mu \tau / 2$ becomes

$$
f_{\tau^{\prime}}^{\prime}+6 f^{\prime 2} f_{\xi}^{\prime}+2 f_{\xi \xi \xi}^{\prime}=-2 \frac{\alpha J}{\mu} f_{\xi \xi}^{\prime},
$$

where $J=4\left(\mu A / \mu_{0} v k\right)$. The term proportional to $\alpha$ in (8) leads to slow variation of soliton parameters and distortion of the soliton shape [1]. The one-soliton solution of this perturbed $\mathrm{mKdV}$ is of the form $f^{\prime}=2 g_{0}\left(\tau^{\prime}\right)\left[\operatorname{sech}(z)-W\left(z, \tau^{\prime}\right)\right]$, where $z=2 g_{0}\left(\tau^{\prime}\right)\left[\xi-\phi_{0}\left(\tau^{\prime}\right)\right]$ and the parameters $g_{0}\left(\tau^{\prime}\right)$ and $\phi_{0}\left(\tau^{\prime}\right)$ are found from the relations

$$
\frac{d g_{0}}{d \tau^{\prime}}=\frac{1}{2} \int_{-\infty}^{\infty} R \frac{d z}{\cosh z} \text { and } \frac{d \phi_{0}}{d \tau^{\prime}}=4 g_{0}^{2}+\frac{1}{4 g_{0}^{2}} \int_{-\infty}^{\infty} R \frac{z d z}{\cosh z} .
$$




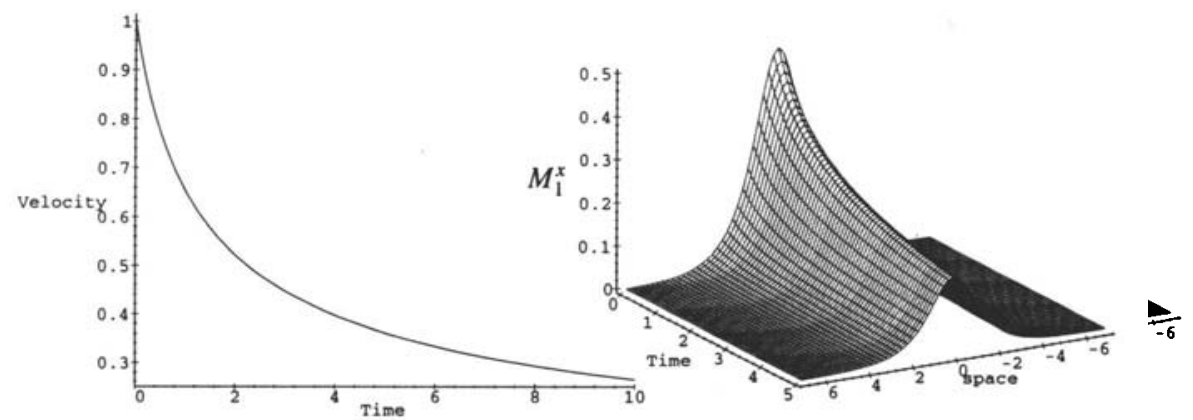

(a)

(b)

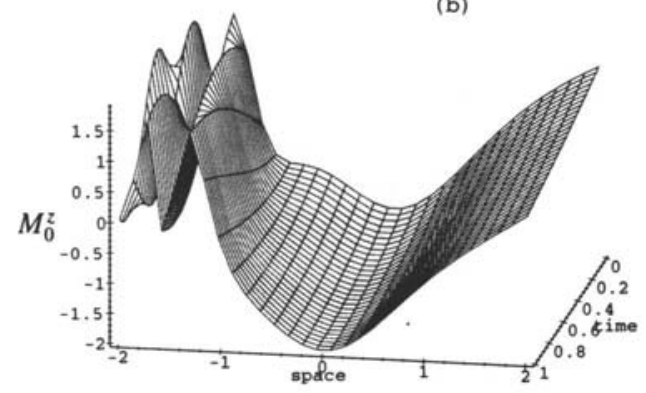

(c)

FIGURE 2. (a) Deceleration of the electromagnetic soliton for $\alpha=1.0, J / \mu=-1 / 4$ and $u(0)=1$; (b) damping of the magnetization soliton $\left(M_{1}^{x}\right)$; and (c) distortion of the magnetization soliton $\left(M_{0}^{z}\right)$ in a charged ferromagnetic medium.

The correction to the soliton $W\left(z, \tau^{\prime}\right)$ is determined from a cumbersome expression which has the asymptotic form $W=\left(z^{2} e^{-z} / 32 g_{0}^{4}\right) \int_{-\infty}^{\infty}(R / \cosh z) d z, z \rightarrow \infty$, and $W=\left(2 \sigma^{\prime} z / 32 g_{0}^{4}\right) \int_{-\infty}^{\infty} R d z, z \rightarrow-\infty$, where $1 / \sigma^{\prime}=8 \int g_{0}^{2} d \tau^{\prime}$. Here $R$ stands for the right-hand side of the perturbed mKdV equation. The above results give the variation of soliton shape and we observe that the effect of Gilbert damping leads to (i) the deceleration of the electromagnetic soliton, which is illustrated in Figure 2 (a), (ii) damping of the magnetization soliton which is shown in Figure 2 (b) and (iii) the distortion of the magnetization soliton at one end which is illustrated in Figure 2 (c).

\section{An electromagnetic soliton in an anisotropic ferromagnetic medium}

A. Linear medium In nature, not all ferromagnetic materials are isotropic. The spinorbit coupling of the ferromagnetic ion in the medium may interact with the crystal field of diamagnetic ions thus creating an easy axis or easy-plane-type anisotropy. The anisotropy of the medium due to the crystal field effect can be introduced by inducting a term of the form $-2 \beta M^{x} \mathbf{n}$ to the effective field where $\beta$ is the anisotropy parameter 
and $\mathbf{n}=(1,0,0)$. The undamped LL equation $((1)$ when $\alpha=0)$ in this case takes the form

$$
\mathbf{M}_{t}=\mathbf{M} \wedge\left[\hat{J} \nabla^{2} \mathbf{M}+2 A \mathbf{H}-2 \beta M^{x} \mathbf{n}\right], \quad \mathbf{M}^{2}=1
$$

Here the anisotropic axis (easy axis) is chosen to be along the direction of propagation of the EMW ( $x$-direction). The set of coupled equations (9) and (5) explain the EMW propagation in an anisotropic ferromagnetic medium. Now we solve them after stretching the wave variable to the same extent as in the previous cases and the time variable by $\tau=\varepsilon^{2} t$. Also, we rescale $\hat{J}$ and $\beta$ as $\hat{J} \rightarrow \varepsilon \hat{J}$ and $2 \beta \rightarrow \varepsilon^{-1} \beta$. Due to the anisotropic nature of the medium here we nonuniformly perturb [13] the magnetization as $M^{x}=M_{0}+\varepsilon M_{1}^{x}+\varepsilon^{2} M_{2}^{x}+\cdots, M^{y}=\varepsilon^{1 / 2}\left[M_{1}^{y}+\varepsilon M_{2}^{y}+\cdots\right]$, $M^{z}=\varepsilon^{1 / 2}\left[M_{1}^{z}+\varepsilon M_{2}^{z}+\cdots\right]$, and also the magnetic induction in the same way. Now we solve the coupled equations (9) and (5) in one dimension using the same perturbation analysis. At $O\left(\varepsilon^{0}\right)$, we obtain the relation $B_{1}^{\alpha}=k M_{1}^{\alpha}$, where $k \equiv$ $\left(B_{0} / M_{0}\right)=\left[\epsilon_{0}\left(c^{2}-v^{2}\right)\right]^{-1}$. At $O\left(\varepsilon^{1}\right)$, after using the results of $O\left(\varepsilon^{0}\right)$, we finally obtain $B_{1}^{x}=0$ and

$$
\psi_{\tau}-\psi_{X X X}++\gamma\left[|\psi|^{2} \psi\right]_{X}=0
$$

where $\psi=\left(M_{1}^{y}-i M_{1}^{z}\right),|\psi|^{2}=M_{1}^{x}, \gamma=-2 A k / \hat{J} M_{0} \mu_{0}$ and $\tau$ is rescaled as $\left(4 A \epsilon_{0} v k^{2} / \hat{J} \mu_{0}\right) \tau$. While writing (10) we have used the transformation $X=\xi+\beta / \hat{J}$ and considered $v / M_{0} \ll \hat{J}$. The $N$-soliton solution of (10) can be obtained using Hirota's bilinearisation procedure [3]. Using the same procedure it is found that the one-soliton solution [9] of (10) takes the form

$$
\psi=G \rho \operatorname{sech}\left[G\left(X+G^{2} \tau\right)+\delta\right]
$$

where

$$
\rho=\left[\frac{-2 \exp \left(2 \eta_{0}\right)}{\gamma \exp \left(\eta_{0}+\eta_{0}^{*}\right)}\right]^{1 / 2} \text { and } \delta=\frac{1}{2} \ln \left[\frac{-\gamma \exp \left(\eta_{0}+\eta_{0}^{*}\right)}{8 G^{2}}\right] .
$$

Here $\eta_{0}$ is a complex constant and $G$ is a real parameter used in Hirota's bilinearisation technique. Further, the $N$-soliton solution can also be constructed as done in [9]. Using (11) in the relation for $\psi$ and $|\psi|^{2}$ and also in the relation connecting the magnetic induction and the magnetization, we obtain the components of magnetic induction and the magnetic field. The above results show that the excitation of magnetization, the magnetic induction and hence the magnetic field of the EMW are highly localized and appear in the form of solitons.

B. Nonlinear medium Ferromagnetic materials when treated show a nonlinear relationship between magnetic induction and the magnetic field which is represented 
by $\mathbf{H}=\left[\mathbf{B}+\mathbf{B}_{N L}\right] / \mu_{0}-\mathbf{M}$, where $\mathbf{B}_{N L}$ is the nonlinear component of the magnetic induction. For our analysis we choose the nonlinear part of the magnetic induction $\mathbf{B}_{N L}$ in terms of the magnetization of the ferromagnetic medium by writing $\mathbf{B}_{N L}=-u \mu_{0} \int \mathbf{M} \cdot[\nabla \cdot \mathbf{M}] d \vec{x}$. Using this the LL equation can be written as

$$
\mathbf{M}_{i}=\mathbf{M} \wedge\left[\hat{J} \nabla^{2} \mathbf{M}+\frac{2 A}{\mu_{0}}\left(\mathbf{B}-u \mu_{0} \int \mathbf{M} \cdot[\nabla \cdot \mathbf{M}] d \vec{x}\right)-2 \beta M^{x} \mathbf{n}\right], \quad \mathbf{M}^{2}=1 .
$$

Similarly, using the nonlinear relation in (3) and solving it with the LL equation (12) as done in the linear anisotropic case [13] at $O\left(\varepsilon^{1}\right)$ by making the transformation $\psi(\eta, \hat{t})=q(\eta, \hat{t}) e^{i(-\eta+\hat{i} / 18) / 6}$ where $\eta=\xi+\tau / 18$ and $\hat{t}=\tau$ and after rescaling $\tau$ as as $-\left(4 A \epsilon_{0} k^{2} v / \hat{J} \mu_{0}\right) \tau$, we finally obtain

$$
i q_{\hat{\imath}}+\frac{1}{2} q_{\eta \eta}+|q|^{2} q+i\left[q_{\eta \eta}+6|q|^{2} q_{\eta}+3\left(|q|^{2}\right)_{\eta} q\right]=0 .
$$

While writing (13) we have chosen $A k=3 \hat{J} \mu_{0} M_{0}, \beta=\hat{J} / 36, u=-1 / 2 M_{0}$ and assumed $v / M_{0} \ll \hat{J}$. Equation (13) is the well-known integrable nonlinear evolution equation proposed by Sasa and Satsuma [10] while studying the integrability aspects of generalised nonlinear Schrödinger equations and the $N$-soliton solutions were obtained by them using the inverse scattering transform method after finding the Lax pair. Equation (13) also describes the propagation of optical pulses through dielectric fibre medium when the higher order effect of the medium, namely the Raman process, is taken into account [8]. For example, the one-soliton solution of (13) can be written as [10]

$$
q(\eta, \hat{t})=\frac{\kappa_{1} e^{i F}\left[2 \cosh G+(L-1) e^{-G}\right]}{|L|+\cosh [2 G-\log |L|]}
$$

where

$$
\begin{aligned}
G & =\kappa_{1}\left[\eta-\left\{\kappa_{2}-\left(\kappa_{1}^{2}-3 \kappa_{2}^{2}\right)\right\} \hat{t}-\eta^{(0)}\right], \\
F & =\kappa_{2}\left[\eta+\left\{\left(\kappa_{1}^{2}-\kappa_{2}^{2}\right) / 2 \kappa_{2}+\left(\kappa_{2}^{2}-3 \kappa_{1}^{2}\right)\right\} \hat{t}-\eta^{(1)}\right], \\
L & =1-\frac{6 i \kappa_{1}}{\left(6 \kappa_{2}-1\right)} .
\end{aligned}
$$

Here $\kappa_{2}=\kappa_{0}+1 / 6, \eta^{(1)}=\kappa_{0} \hat{\eta}^{(1)} / \kappa$ and $\kappa_{0}$ and $\kappa_{1}$ are real parameters and $\eta^{(0)}$ and $\hat{\eta}^{(1)}$ are real phase constants used in the inverse scattering technique. As before using the above one-soliton solution and successive transformations we obtain the onesoliton solution for $\psi$ and finally the components of magnetic induction and hence the components of the magnetic field. This clearly shows that the nonlinearity of the medium has modulated the magnetic field component of the EMW in the form of a soliton. 


\section{Conclusions}

We have found that the propagation of EMWs in a charge-free isotropic ferromagnetic medium is governed by soliton modes. While soliton excitations of the magnetic induction are restricted to the plane normal to the direction of propagation, the magnetic field is restricted to the normal plane at the leading order of perturbation and comes out at higher orders during propagation. However, when free charges are added to the isotropic medium the electromagnetic soliton decelerates, the amplitude of the soliton decreases and the shape of the soliton is distorted. Also when the EMW propagates in a charge-free anisotropic (linear and nonlinear) ferromagnetic medium, the magnetic field component of the electromagnetic field and the magnetization of the medium are identified in the form of solitons.

Acknowledgements The work of M. D. forms part of a major DST project. V. V. acknowledges CSIR, India for financial support in the form of a Senior Research Fellowship.

\section{References}

[1] F. G. Bass and N. N. Nasonov, "Nonlinear electromagnetic-spin waves", Phys. Reps. 4 (1990) 165-223.

[2] M. Daniel, V. Veerakumar and R. Amuda, "Soliton and electromagnetic wave propagation in ferromagnetic medium", Phys. Rev. E55 (1997) 3619-3623.

[3] R. Hirota, "Exact $N$-soliton solution of the wave equation of long waves in shallow water and in nonlinear lattices", J. Math. Phys. 14 (1973) 810-814.

[4] J. D. Jackson, Classical Electrodynamics (Wiley Eastern, New York, 1978).

[5] L. Landau and E. Lifshitz, "On the theory of the dispersion of magnetic permeability in ferromagnetic bodies", Phys. Z. Sowjetunion 8 (1935) 153-166.

[6] H. Leblond, "Electromagnetic waves in ferrites: from linear absorption to the nonlinear Schrödinger equation", J. Phys. A. 29 (1996) 4623-4639.

[7] I. Nakata, "Nonlinear electromagnetic waves in ferromagnets", J. Phys. Soc. Japan 60 (1991) $77-81$.

[8] K. Porsezian, M. Daniel and M. Lakshmanan, "Integrability aspects of a higher order nonlinear Schrödinger equation in nonlinear optics", in Nonlinear Evolution Equations and Dynamical Systems (eds. V. G. Makhankov, I. Puzynin and G. Pasheev), (World Scientific, Singapore, 1993) $436-443$.

[9] R. Radhakrishnan and M. Lakshmanan, "Exact soliton solutions to coupled nonlinear Schrödinger equation with higher-order effects", Phys. Rev. E54 (1996) 2949-2955.

[10] N. Sasa and J. Satsuma, "New-type of soliton solutions for higher-order nonlinear Schrödinger equations", J. Phys. Soc. Japan 66 (1991) 409-417.

[11] J. L. Simonds, "Magnetoelectronics today and tomorrow", Phys. Today 48 (1995) 26-32.

[12] V. Veerakumar and M. Daniel, "Electromagnetic soliton damping in a ferromagnetic medium", Phys. Rev. E57 (1998) 1197-1200.

[13] V. Veerakumar and M. Daniel, "Electromagnetic soliton in an anisotropic ferromagnetic medium under nonuniform perturbation", Phys. Letts. A278 (2001) 331-338.

[14] M. Wadati, "The modified Korteweg-de Vries equation", J. Phys. Soc. Japan 32 (1972) $1681 \mathrm{ff}$. 